PROCEEDINGS OF THE

AMERICAN MATHEMATICAL SOCIETY

Volume 133, Number 8, Pages 2233-2235

S 0002-9939(05)07967-0

Article electronically published on March 17, 2005

\title{
A PURE SUBALGEBRA OF A FINITELY GENERATED ALGEBRA IS FINITELY GENERATED
}

\author{
MITSUYASU HASHIMOTO
}

(Communicated by Bernd Ulrich)

\begin{abstract}
We prove the following. Let $R$ be a Noetherian commutative ring, $B$ a finitely generated $R$-algebra, and $A$ a pure $R$-subalgebra of $B$. Then $A$ is finitely generated over $R$.
\end{abstract}

In this paper, all rings are commutative. Let $A$ be a ring and $B$ an $A$-algebra. We say that $A \rightarrow B$ is pure, or $A$ is a pure subring of $B$, if for any $A$-module $M$, the map $M=M \otimes_{A} A \rightarrow M \otimes_{A} B$ is injective. Considering the case $M=A / I$, where $I$ is an ideal of $A$, we immediately have that $I B \cap A=I$.

There have been a number of cases where it has been shown that if $B$ has a good property and $A$ is a pure subring of $B$, then $A$ has a good property. If $B$ is a regular Noetherian ring containing a field, then $A$ is Cohen-Macaulay [5], 4]. If $k$ is a field of characteristic zero, $A$ and $B$ are essentially of finite type over $k$, and $B$ has at most rational singularities, then $A$ has at most rational singularities [1].

In this paper, we prove the following.

Theorem 1. Let $R$ be a Noetherian ring, $B$ a finitely generated $R$-algebra, and $A$ a pure $R$-subalgebra of $B$. Then $A$ is finitely generated over $R$.

The case that $B$ is $A$-flat is proved in [3, Corollary 2.6]. This theorem is on the same line as the finite generation results in 3 .

To prove the theorem, we need the following, which is a special case of a theorem of Raynaud-Gruson [7, 8].

Theorem 2. Let $A \rightarrow B$ be a homomorphism of Noetherian rings, and $\varphi: X \rightarrow Y$ the associated morphism of affine schemes. Let $U \subset Y$ be an open subset, and assume that $\varphi: \varphi^{-1}(U) \rightarrow U$ is flat. Then there exists some ideal $I$ of $A$ such that $V(I) \cap U=\emptyset$, and such that the morphism $\Phi: \operatorname{Proj} R_{B}(B I) \rightarrow \operatorname{Proj} R_{A}(I)$, determined by the associated morphism of the Rees algebras $R_{A}(I):=A[t I] \rightarrow$ $R_{B}(B I):=B[t B I]$, is flat.

The morphism $\Phi$ in the theorem is called a flattening of $\varphi$.

Proof of Theorem 1. Note that for any $A$-algebra $A^{\prime}$, the homomorphism $A^{\prime} \rightarrow$ $B \otimes_{A} A^{\prime}$ is pure.

Received by the editors December 30, 2003.

2000 Mathematics Subject Classification. Primary 13E15.

Key words and phrases. Pure subalgebra, finite generation, flattening.

(C)2005 American Mathematical Society Reverts to public domain 28 years from publication 
Since $B$ is finitely generated over $R$, it is Noetherian. Since $A$ is a pure subring of $B, A$ is also Noetherian. So if $A_{\text {red }}$ is finitely generated, then so is $A$. Replacing $A$ by $A_{\text {red }}$ and $B$ by $B \otimes_{A} A_{\text {red }}$, we may assume that $A$ is reduced.

Since $A \rightarrow \prod_{P \in \operatorname{Min}(A)} A / P$ is finite and injective, it suffices to prove that each $A / P$ is finitely generated for $P \in \operatorname{Min}(A)$, where $\operatorname{Min}(A)$ denotes the set of minimal primes of $A$. By the base change, we may assume that $A$ is a domain.

There exists some minimal prime $P$ of $B$ such that $P \cap A=0$. Assume the contrary. Then take $a_{P} \in P \cap A \backslash\{0\}$ for each $P \in \operatorname{Min}(B)$. Then $\prod_{P} a_{P}$ must be nilpotent, which contradicts our assumption that $A$ is a domain.

So by [6, (2.11) and (2.20)], $A$ is a finitely generated $R$-algebra if and only if $A_{\mathfrak{p}}$ is a finitely generated $R_{\mathfrak{p}}$-algebra for each $\mathfrak{p} \in \operatorname{Spec} R$. So we may assume that $R$ is a local ring.

By the descent argument [2, (2.7.1)], $\hat{R} \otimes_{R} A$ is a finitely generated $\hat{R}$-algebra if and only if $A$ is a finitely generated $R$-algebra, where $\hat{R}$ is the completion of $R$. So we may assume that $R$ is a complete local ring. We may lose the assumption that $A$ is a domain (even if $A$ is a domain, $\hat{R} \otimes_{R} A$ may not be a domain). However, doing the same reduction argument as above if necessary, we may still assume that $A$ is a domain.

Let $\varphi: X \rightarrow Y$ be a morphism of affine schemes associated with the map $A \rightarrow B$. Note that $\varphi$ is a morphism of finite type between Noetherian schemes. We denote the flat locus of $\varphi$ by Flat $(\varphi)$. Then $\varphi(X \backslash$ Flat $(\varphi))$ is a constructible set of $Y$ not containing the generic point. So $U=Y \backslash \overline{\varphi(X \backslash \text { Flat }(\varphi))}$ is a dense open subset of $Y$, and $\varphi: \varphi^{-1}(U) \rightarrow U$ is flat. By Theorem [2 there exists some nonzero ideal $I$ of $A$ such that $\Phi: \operatorname{Proj} R_{B}(B I) \rightarrow \operatorname{Proj} R_{A}(I)$ is flat.

If $J$ is a homogeneous ideal of $R_{A}(I)$, then $J$ can be expressed as $J=\bigoplus_{n \geq 0} J_{n} t^{n}$ $\left(J_{n} \subset I^{n}\right)$. Since $A$ is a pure subalgebra of $B$, we have $J_{n} B \cap I^{n}=J_{n}$ for each $n$. Since $J R_{B}(B I)=\bigoplus_{n \geq 0}\left(J_{n} B\right) t^{n}$, we have that $J R_{B}(B I) \cap R_{A}(I)=J$. Namely, any homogeneous ideal of $R_{A}(I)$ is contracted from $R_{B}(B I)$.

Let $P$ be a homogeneous prime ideal of $R_{A}(I)$. Then there exists some minimal prime $Q$ of $P R_{B}(B I)$ such that $Q \cap R_{A}(A I)=P$. Assume the contrary. Then for each minimal prime $Q$ of $P R_{B}(B I)$, there exists some $a_{Q} \in\left(Q \cap R_{A}(A I)\right) \backslash P$. Then $\prod a_{Q} \in \sqrt{P R_{B}(B I)} \cap R_{A}(A I) \backslash P$. However, we have

$$
\sqrt{P R_{B}(B I)} \cap R_{A}(I)=\sqrt{P R_{B}(B I) \cap R_{A}(I)}=\sqrt{P}=P,
$$

and this is a contradiction. Hence $\Phi: \operatorname{Proj} R_{B}(B I) \rightarrow \operatorname{Proj} R_{A}(I)$ is faithfully flat.

Since $\operatorname{Proj} R_{B}(B I)$ is of finite type over $R$ and $\Phi$ is faithfully flat, we have that $\operatorname{Proj} R_{A}(I)$ is of finite type by [3, Corollary 2.6]. Note that the blow-up $\operatorname{Proj} R_{A}(I) \rightarrow Y$ is proper surjective. Since $R$ is excellent, $Y$ is of finite type over $R$ by [3, Theorem 4.2]. Namely, $A$ is a finitely generated $R$-algebra.

\section{REFERENCES}

[1] J.-F. Boutot, Singularités rationelles et quotients par les groupes réductifs, Invent. Math. 88 (1987), 65-68. MR0877006 (88a:14005)

[2] A. Grothendieck, Eléments de Géométrie Algébrique IV, IHES Publ. Math. 20 (1964), 24 (1965), 28 (1966), 32 (1967). MR0173675(30:3885); MR0199181 (33:7330), MR0217086 $(36: 178) ;$ MF $0238860(39: 220)$

[3] M. Hashimoto, "Geometric quotients are algebraic schemes" based on Fogarty's idea, J. Math. Kyoto Univ. 43 (2003), 807-814. MR2030799 (2004j:14050) 
[4] M. Hochster and C. Huneke, Applications of the existence of big Cohen-Macaulay algebras, Adv. Math. 113 (1995), 45-117. MK1332808 (96d:13014)

[5] M. Hochster and J. L. Roberts, Rings of invariants of reductive groups acting on regular rings are Cohen-Macaulay, Adv. Math. 13 (1974), 115-175. MR.0347810 (50:311)

[6] N. Onoda, Subrings of finitely generated rings over a pseudo-geometric ring, Japan. J. Math. 10 (1984), 29-53. MR0884429 (88d:13024)

[7] M. Raynaud, Flat modules in algebraic geometry, Compositio Math. 24 (1972), 11-31. MR 0302645 (46:1789)

[8] M. Raynaud and L. Gruson, Critères de platitude et de projectivité. Techniques de "platification" d'un module, Invent. Math. 13 (1971), 1-89. MR0308104 (46:7219)

Graduate School of Mathematics, Nagoya University, Chikusa-ku, Nagoya 464-8602, JAPAN

E-mail address: hasimoto@math.nagoya-u.ac.jp 